CARDIOVASCULAR MEDICINE

\title{
Long term risk stratification of patients with acute coronary syndromes: characteristics of troponin T testing and continuous ST segment monitoring
}

\author{
B L Nørgaard, K Andersen, K Thygesen, J Ravkilde, P Abrahamsson, L Grip, M Dellborg
}

Heart 2004;90:739-744. doi: 10.1136/hrt.2003.020479

See end of article for authors' affiliations

Correspondence to:

Dr Bjarne L Nørgaard,

Department of Cardiology

B, Aarhus University

Hospital, Skejby Hospital

Brendstrupgaardsvej 100,

8200 Aarhus N, Denmark;

bnorgaard@dadlnet.dk

Accepted 16 January 2004
Objective: To examine the long term prognostic characteristics of troponin T testing and continuous multilead ST segment monitoring in combination with clinical and 12 lead ECG risk indicators in patients with acute coronary syndromes (ACS).

Patients and design: Patients with suspected ACS ( $n=213$ ) were studied. Troponin T was analysed in blood samples collected during the first 12 hours after admission. Continuous vectorcardiography ST segment monitoring was performed for 24 hours and the number of ST vector magnitude episodes was registered. Patients were followed up for a median of 28 months. The end point was a composite of cardiac death and acute myocardial infarction.

Results: Thirty eight (18\%) patients reached the composite end point. The median (interquartile range) time from study inclusion to the time of the composite end point was longer for patients predicted to be at risk by troponin T testing $(\mathrm{n}=27)$ than for those predicted to be at risk by ST segment monitoring $(\mathrm{n}=20)(8.4$ $(0.2-15)$ months $v 0.3(0.1-4.3)$ months, $p=0.04)$. Significant univariate predictors of the composite end point were age $\geqslant 65$ years, diabetes, previous myocardial infarction, congestive heart failure, use of $\beta$ blockers or diuretics at admission, 12 lead ECG ST segment depression at admission, troponin T concentration $\geqslant 0.10 \mu \mathrm{g} / \mathrm{l}$, and $\geqslant 1$ ST vector magnitude episodes. Age $\geqslant 65$ years, previous myocardial infarction, and troponin $\mathrm{T}$ concentration $\geqslant 0.10 \mu \mathrm{g} / \mathrm{l}$ provided independent prognostic information after multivariate analysis of potential risk variables. The prognostic value of transient ischaemic episodes in ACS seems to be confined to the short term.

Conclusions: Both biochemical and continuous ECG markers reflect an increased risk for patients with ACS; however, the methods exhibit different temporal risk characteristics.
B oth evaluation of troponin concentrations and continuous ST segment monitoring are valuable tools for early risk assessment of patients with acute coronary syndromes (ACS) ${ }^{1-16}$ Both troponin $\mathrm{T}$ and continuous multi-lead ST segment monitoring have independent short term prognostic value in ACS. ${ }^{15}$ However, no previous study has assessed the long term prognostic significance of combining troponin $\mathrm{T}$ testing and continuous ST segment monitoring among such patients. We have extended the follow up period in the TRIM (thrombin inhibition in myocardial ischemia) trial from 30 days to a medium of 28 months and investigated the long term prognostic significance of early troponin $\mathrm{T}$ testing and continuous ST segment monitoring in combination with clinical and 12 lead ECG risk indicators among patients with ACS.

\section{PATIENTS AND METHODS}

\section{Study population}

The study population was composed of patients with suspected ACS enrolled in the TRIM trial $(\mathrm{n}=1209)$, a prospective, randomised, multicentre trial investigating the efficacy of a specific thrombin inhibitor (inogatran) in comparison with heparin. ${ }^{17}$ The study design and primary results have been published. ${ }^{17}$ Briefly, patients suspected of having unstable angina (new onset of presumably ischaemic chest pain, deterioration of previously stable angina, or onset of angina at rest) with angina $<24$ hours before study inclusion were eligible. The clinical diagnosis had to be supported by one of the following: ECG changes compatible with myocardial ischaemia-that is, ST depression or T wave inversion ( $\geqslant 0.1 \mathrm{mV}$ in at least two contiguous leads); or a history of previous myocardial infarction, positive coronary angiography, positive myocardial scintigraphy, or a positive exercise test. The main exclusion criteria were an indication for thrombolysis or any other condition considered to increase the risk of bleeding.

Patients were treated with a three day intravenous infusion of heparin or one of three doses of inogatran. ${ }^{17}$ All patients received low dose aspirin if tolerated. Use of other medications such as nitrates, $\beta$ blockers, and calcium antagonists, as well as referral for coronary angiography, angioplasty, or artery bypass grafting, was left to the discretion of the treating physician.

Two hundred and thirty two patients provided blood samples for analysis of troponin $\mathrm{T}$ and underwent ST segment monitoring. Blood samples were missing for one patient. Thirteen patients were prospectively excluded from ST segment analysis because of the presence of bundle branch block, pacemaker in situ, or $<16$ hours ST segment monitoring recording time. In five patients ST segment analysis could not be performed because of technical problems. The remaining 213 patients form the basis of this report.

Written informed consent was obtained from all patients. The protocol was approved by the regional scientific ethical committees of the participating centres.

Abbreviations: ACS, acute coronary syndromes; $A M I$, acute myocardial infarction; $\mathrm{Cl}$, confidence interval; $H R$, hazard ratio; IQR, interquartile range; ST-VM, ST vector magnitude; TRIM, thrombin inhibition in myocardial ischemia 


\section{Twelve lead ECG}

A 12 lead ECG was obtained at admission. ECGs were interpreted by the local investigators and categorised according to the predominant ST-T segment status-that is, whether ST elevation, ST depression, $\mathrm{T}$ wave inversion, or any ST-T wave change were present.

\section{Troponin T}

Blood specimens for troponin $\mathrm{T}$ analysis were obtained at inclusion and after six and 12 hours. Plasma was kept at $-80^{\circ} \mathrm{C}$ and sent to a core laboratory for analysis by personnel without knowledge of the patients' status (Aarhus University Hospital, Aarhus, Denmark). Troponin $\mathrm{T}$ was measured with an immunoassay (ELISA troponin(e) T) on the ES 300 analyser (Boehringer Mannheim GmbH, Mannheim, Germany). The method has been described in detail elsewhere ${ }^{18}$ A troponin $\mathrm{T}$ concentration of $0.10 \mu \mathrm{g} / \mathrm{l}$ during the first 12 hour period was used to differentiate between high and low risk patients. ${ }^{136716}$

\section{ST segment monitoring}

Continuous vectorcardiography ST segment monitoring was performed for 24 hours after inclusion with the MIDA 1000 or Coronet system (Ortivus Medical AB, Täby, Sweden). The method of ischaemia monitoring by vectorcardiography has previously been described in detail. ${ }^{12} 1315$ Briefly, ECG complexes are continuously collected, sampled for one minute periods, and averaged into three orthogonal leads, $\mathrm{X}, \mathrm{Y}$, and Z. ST vector magnitude (ST-VM) is defined as the summarised deviation of the ST segment from the isoelectric level measured $20 \mathrm{~ms}$ after the $\mathrm{J}$ point. At the end of each averaging period, ST-VM is calculated and presented as a trend curve updated continuously during the whole recording period. Recordings were stored on floppy disks and sent for core laboratory analysis by personnel without knowledge of the patients' status (Sahlgrenska University Hospital, Östra, Gothenburg, Sweden). The presence of transient myocardial ischaemia in the present study was defined as $\geqslant 1$ ST-VM episodes because this algorithm is easy to use in the clinical setting and its accuracy in discriminating between high and low risk patients with ACS has been documented as high. ${ }^{12} 1315$

\section{Follow up}

The end point was a composite of the events cardiac death and acute myocardial infarction (AMI) occurring within the follow up period. Cardiac adverse events occurring during hospitalisation and up to 30 days after the index event were evaluated by an independent end point committee. Thereafter, information about adverse events was obtained from hospital records and registers. Median (interquartile range (IQR), range) follow up time for all patients was 28 (14-31, 0.03-52) months and median (IQR, range) follow up of event-free patients was 29 (14-32, 12-52) months. AMI was considered present when it occurred at least six hours after randomisation and when two of the following criteria were met: typical anginal chest pain lasting at least 20 minutes; typical ECG changes indicative of AMI (new Q waves or ST elevation followed by T wave inversion in at least two leads) in two consecutive recordings; and typical increase of cardiac biochemical markers on two occasions and occurring separately from any increase taking place at inclusion.

\section{Statistical analyses}

Data were analysed on an intention to treat basis. Owing to non-normal distribution, troponin $\mathrm{T}$ concentration and ST segment monitoring data are presented as medians (IQR, range). Group continuous variables were compared by the
Mann-Whitney U test and categorical variables were compared by the $\chi^{2}$ or Fisher's exact test as appropriate. Univariate predictors of the composite end point were identified by the log rank test. Survival curves were constructed by the Kaplan-Meier method. Cox regression trend analysis was used to explore the significance of the number of ST-VM episodes in relation to the troponin $\mathrm{T}$ concentration and to the probability of the composite end point. Forward stepwise Cox regression analysis was used to detect independent predictors of the composite end point with variables of potential significance $(p<0.10)$ identified in the univariate analysis. A probability value of $p<0.20$ was the criterion for selection. At first, baseline variables of potential significance were evaluated (model I). Once these were accounted for, the added value of information from troponin $\mathrm{T}$ testing and from ST segment monitoring was explored (model II). To evaluate the potential influence on the clinical outcome of coronary revascularisations (angioplasty or coronary artery bypass grafting) performed during follow up, statistical analyses were repeated after censoring of patients at the time of revascularisation. Results are expressed as hazard ratios (HR) with 95\% confidence intervals (CI) and p values. A probability value of $p<0.05$ was considered significant. Data were analysed with the SAS statistical package (version 8.1, SAS Institute, Cary, North Carolina, USA).

\section{RESULTS}

\section{General findings}

In $92(43 \%)$ patients troponin T concentration was $\geqslant 0.10 \mu \mathrm{g} / \mathrm{l}$ (median $0.52 \mu \mathrm{g} / \mathrm{l}$, IQR 0.20-1.90 $\mu \mathrm{g} / \mathrm{l}$, range 0.10-3.69 $\mu \mathrm{g} / \mathrm{l}$ ). Admission troponin $\mathrm{T}$ concentration was $\geqslant 0.10 \mu \mathrm{g} / \mathrm{l}$ in 77 (36\%) patients. Troponin $\mathrm{T}$ concentration was $0.00 \mu \mathrm{g} / \mathrm{l}$ in $75(35 \%)$ patients. Sixty nine $(32 \%)$ of the patients had $\geqslant 1$ ST-VM episode (median 3, IQR 1-6, range 1-24). The median time of ST segment monitoring was 24 hours. A positive quantitative relation between the troponin $\mathrm{T}$ concentration and the number of ST-VM episodes was found (fig 1). Table 1 presents baseline clinical and 12 lead ECG characteristics according to the troponin $T$ concentration and presence of ST-VM episodes. Compared with the total study population, patients with ST segment depression on the admission ECG had more ST-VM episodes and patients with increased troponin $\mathrm{T}$ concentration less commonly had previous angina. Otherwise, the two patient groups had similar characteristics and were representative of the main study population. Of nine patients with ST elevation in the admission ECG, three were retrospectively categorised as having ST elevation AMI.

\section{Clinical outcome}

After one month 12 (5.6\%) patients had died or experienced a non-fatal AMI. After one year $22(10 \%)$ and at the end of the study period $38(18 \%)$ patients had died or had a non-fatal AMI. Four (1.9\%) patients died during the study period. Fifty seven $(27 \%)$ of the patients underwent coronary revascularisation within 30 days. At the end of follow up 96 (45\%) patients had undergone coronary revascularisation. No revascularisations were performed in 94 event-free patients and 23 patients who reached the composite end point. Among eight patients who underwent revascularisation before reaching the combined end point, three developed an AMI $<48$ hours after the procedure. In 89 patients (revascularised event-free patients and patients undergoing revascularisation before the adverse event), revascularisation may have influenced the clinical outcome. Among these patients, there was no difference in frequency of revascularisation between patients with $(38 \%)$ and patients without $(45 \%)$ an increased troponin concentration $(p=0.4)$, nor 


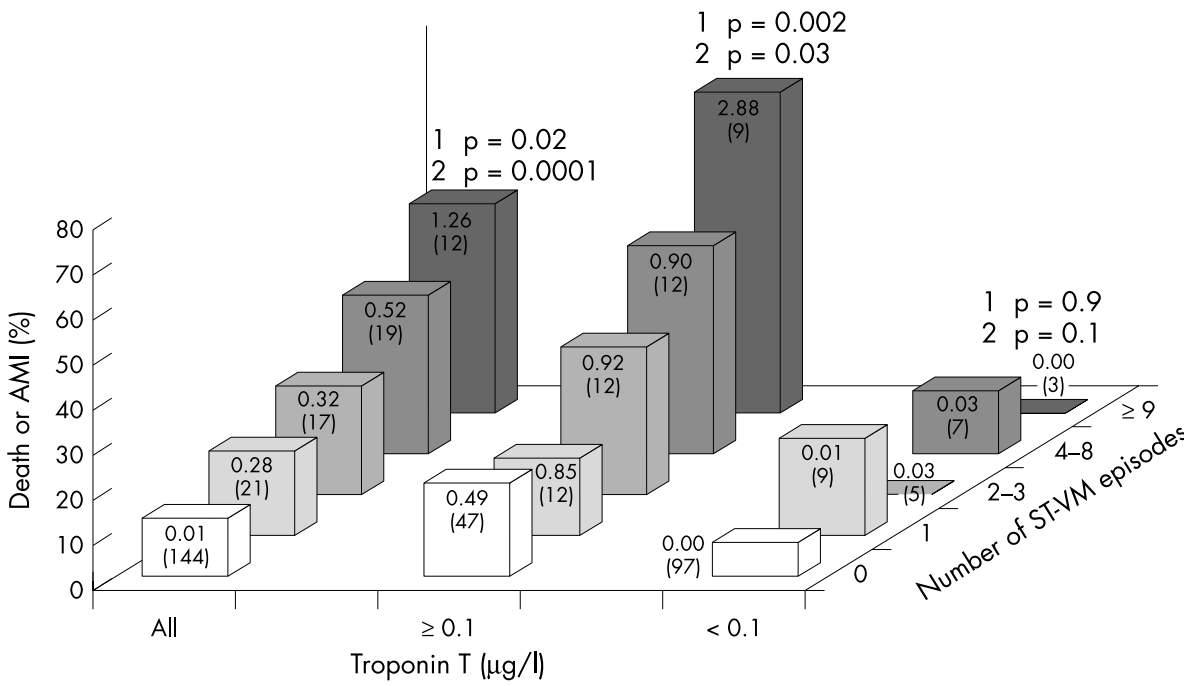

Figure 1 Incidence of cardiac death or non-fatal acute myocardial infarction (AMI) at a median of 28 months' follow up, according to the troponin T concentration and the number of ST-VM episodes. Median troponin T concentration and the number of patients in each group (in parentheses) are shown on the bars. $p$ Values for trend analysis of the number of ST-VM episodes in relation to the probability of death or AMI (1) and the number of STVM episodes in relation to the troponin T concentration (2) are shown.

between patients with (45\%) and patients without (40\%) STVM episodes $(p=0.6)$. Median (IQR, range) time from study inclusion to revascularisation among these patients was $1.5(0.4-7,0.03-24)$ months. In the same patient group, no significant differences in time to revascularisation according to the troponin concentration or the presence of ST-VM episodes were found.

\section{Predictors of cardiac death or AMI}

Significant univariate predictors of the composite end point were age $\geqslant 65$ years, diabetes, previous myocardial infarction, congestive heart failure, use of $\beta$ blockers or diuretics at admission, 12 lead ECG ST segment depression at admission, troponin $\mathrm{T}$ concentration $\geqslant 0.10 \mu \mathrm{g} / \mathrm{l}$, and $\geqslant 1$ ST-VM episodes (table 2). Figure 2 shows the cumulative percentage of patients with event-free survival according to the troponin $\mathrm{T}$ concentration or the presence of ST-VM episodes. Median (IQR, range) time from study inclusion to the time of the composite end point was longer for patients predicted to be at risk by troponin $\mathrm{T}$ testing $(\mathrm{n}=27)$ than for patients predicted to be at risk by ST segment monitoring $(\mathrm{n}=20)$ (8.4 $(0.2-15,0.03-27)$ months $v 0.3 \quad(0.1-4.3,0.03-23)$ months, $\mathrm{p}=0.04)$. Figure 3 shows the cumulative percentage of patients with event-free survival according to the troponin $\mathrm{T}$ concentration in relation to the presence of ST-VM episodes.

Multivariate analysis of baseline variables of potential significance identified age $\geqslant 65$ years, previous myocardial infarction, no previous coronary revascularisation, and congestive heart failure as independent predictors of the composite end point (table 3). With the addition of information on troponin $\mathrm{T}$ concentration and ST segment monitoring, only age $\geqslant 65$ years, previous myocardial infarction, and troponin $\mathrm{T} \geqslant 0.10 \mu \mathrm{g} / \mathrm{l}$ had significant independent prognostic significance.

Significant predictors of the combined end point after censoring of patients (revascularised event-free patients and patients having revascularisation performed before the event) at the time of the revascularisation procedure (statistical model II) were previous myocardial infarction (HR 2.48, 95\%

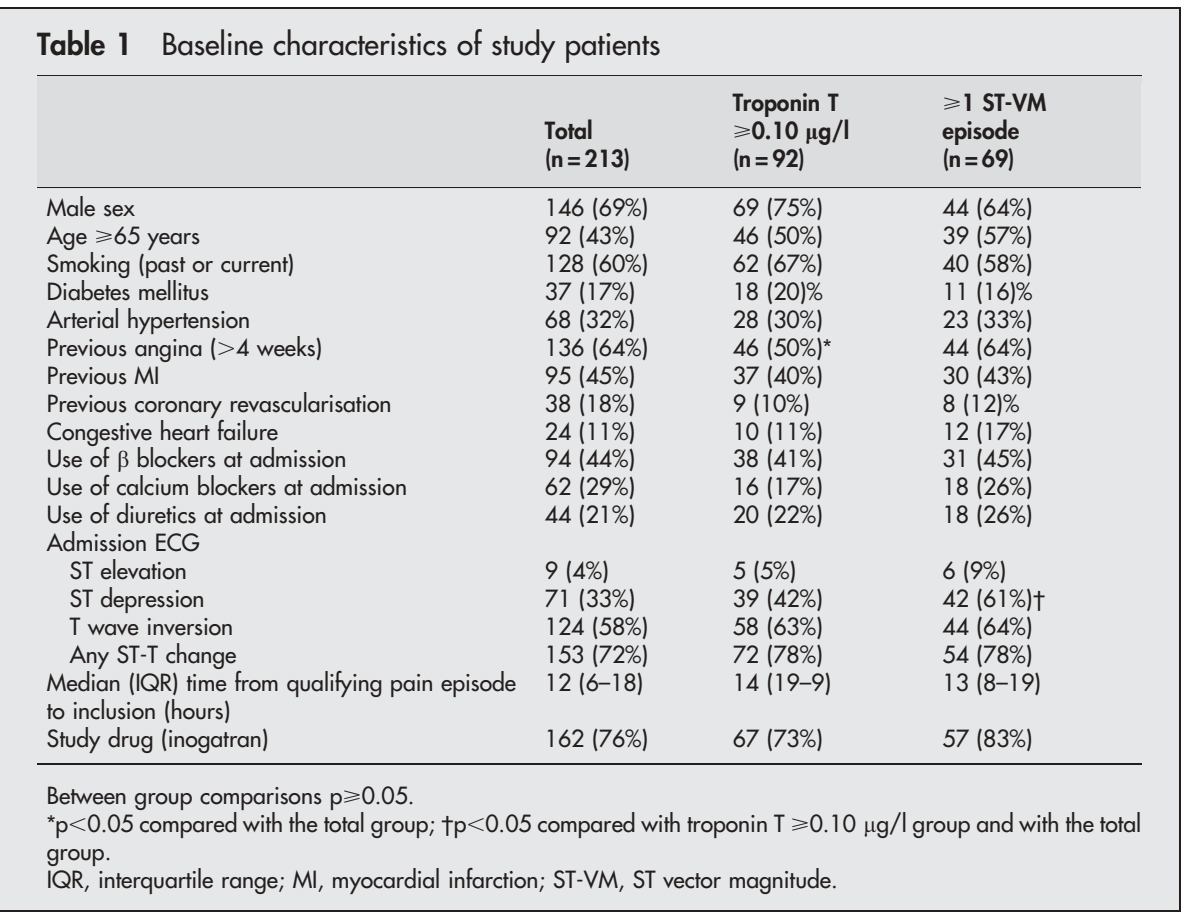




\begin{tabular}{|c|c|c|c|}
\hline & & $\begin{array}{l}\text { Relative frequency } \\
\text { of death or AMI } \\
\text { (\%) }\end{array}$ & p Value \\
\hline Male sex & Yes/No & $16.4 / 20.9$ & 0.41 \\
\hline Age $\geqslant 65$ years & Yes/No & $29.3 / 9.1$ & 0.0002 \\
\hline Smoking (past or current) & Yes/No & $16.4 / 20.0$ & 0.43 \\
\hline Diabetes mellitus & Yes/No & $32.0 / 14.7$ & 0.01 \\
\hline Arterial hypertension & Yes/No & $25.0 / 14.5$ & 0.06 \\
\hline Previous angina ( $>4$ weeks) & Yes/No & $19.1 / 15.6$ & 0.54 \\
\hline Previous MI & Yes/No & $27.2 / 10.7$ & 0.003 \\
\hline Previous revascularisation & Yes/No & $8.0 / 20.0$ & 0.07 \\
\hline Congestive heart failure & Yes/No & $41.7 / 14.8$ & 0.0004 \\
\hline Admission use of $\beta$ blockers & Yes/No & $24.5 / 12.6$ & 0.02 \\
\hline Admission use of calcium & Yes/No & $19.4 / 17.2$ & 0.57 \\
\hline $\begin{array}{l}\text { Admission use of diuretics } \\
\text { Admission ECG }\end{array}$ & Yes/No & $36.4 / 13.0$ & 0.0002 \\
\hline $\begin{array}{l}\text { Admission ECG } \\
\text { ST elevation }\end{array}$ & Yes/No & $22.0 / 17.6$ & 0.67 \\
\hline ST depression & Yes/No & $26.8 / 13.4$ & 0.01 \\
\hline $\mathrm{T}$ wave inversion & Yes/No & $18.5 / 16.9$ & 0.86 \\
\hline Any ST-T change & Yes/No & $19.0 / 15.0$ & 0.55 \\
\hline Troponin $\mathrm{T} \geqslant 0.10 \mu \mathrm{g} / \mathrm{l}$ & Yes/No & $29.3 / 9.1$ & 0.0003 \\
\hline ST-VM $\geqslant 1$ episodes & Yes/No & $29.0 / 12.5$ & 0.0006 \\
\hline Study drug (inogatran) & Yes/No & $19.7 / 11.8$ & 0.16 \\
\hline
\end{tabular}

CI 1.04 to $5.9, \mathrm{p}=0.04$ ), no previous revascularisation (HR $0.30,95 \%$ CI 0.09 to $0.83, \mathrm{p}=0.03$ ), and troponin $\mathrm{T}$ concentration $\geqslant 0.10 \mu \mathrm{g} / \mathrm{l}$ (HR 3.01, 95\% CI 1.24 to 7.40 , $\mathrm{p}=0.015)$. In this subanalysis, the presence of ST-VM
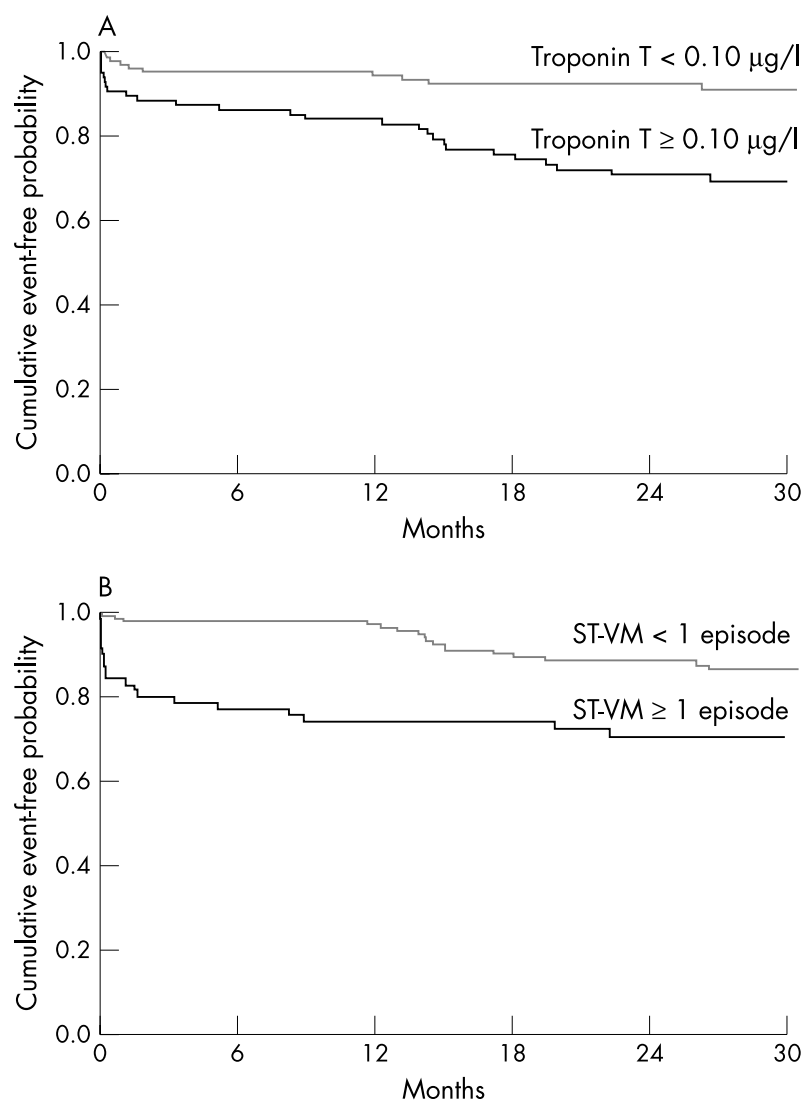

Figure 2 Occurrence of cardiac death or non-fatal AMI at a medium of 28 months' follow up according to troponin T testing and continuous vectorcardiography ST segment monitoring. (A) Event-free probability among patients with or without troponin $T \geqslant 0.10 \mu \mathrm{g} / \mathrm{l}$ (log rank, $p=0.0003$ ). (B) Event-free probability of patients with or without STVM episodes (log rank, $p=0.0006$ ). episodes did not reach significance (HR 1.55, 95\% CI 0.79 to $4.11, \mathrm{p}=0.18$ ).

\section{DISCUSSION}

Admission clinical and 12 lead ECG characteristics, biochemical markers of myocardial necrosis, and continuous ST segment monitoring have proved to be valuable short and long term indicators of worsening outcome after an episode of ACS. ${ }^{1-16}$ However, the present study is the first to investigate the long term prognostic significance of combining troponin $\mathrm{T}$ and ECG monitoring among such patients.

In the present study, troponin $\mathrm{T}$ concentration $\geqslant 0.10 \mu \mathrm{g} / \mathrm{l}$ indicated an almost threefold increase in the risk of a long term unfavourable outcome compared with no troponin $\mathrm{T}$ increase. At 30 days the sensitivity, specificity, and negative predictive value of troponin $\mathrm{T}$ testing in predicting cardiac death or AMI were $67 \%, 58 \%$, and $97 \%$, respectively. At the end of follow up the same measures of accuracy were $71 \%$, $63 \%$, and $91 \%$, respectively. That the risk of a poor outcome among patients who present with ACS and troponin $\mathrm{T}$ increase continues to increase for up to three years is in agreement with previous investigations. ${ }^{2458}$

The sensitivity, specificity, and negative predictive value of one or more ST episodes in predicting a poor outcome at 30 days in this study were $83 \%, 71 \%$, and $99 \%$, respectively. We found a fairly high long term specificity of $72 \%$ and negative predictive value of $88 \%$; however, the sensitivity was only $53 \%$. Thus, the rather low prognostic sensitivity of ECG monitoring in the present study is based on adverse events occurring over the long term. Of those adverse events predicted by ST segment monitoring, 50\% occurred within 30 days after the index event. Beyond this stage it seems that there is no difference in survival rates between patients with and patients without ST episodes. These findings are consistent with a study by Patel and colleagues ${ }^{14}$ of 212 patients with unstable angina showing that the sensitivity of transient ST episodes in predicting death and AMI at six weeks was $54 \%$ (specificity $87 \%$, negative predictive value $97 \%$ ) and $28 \%$ (specificity $88 \%$, negative predictive value $91 \%$ ), respectively, at a median of 31 months' follow up. Overall discrepancies in measures of accuracy between the two studies may be explained by differences in crucial determinants for outcome (that is, study enrolment criteria, treatment protocols, and ECG monitoring techniques).

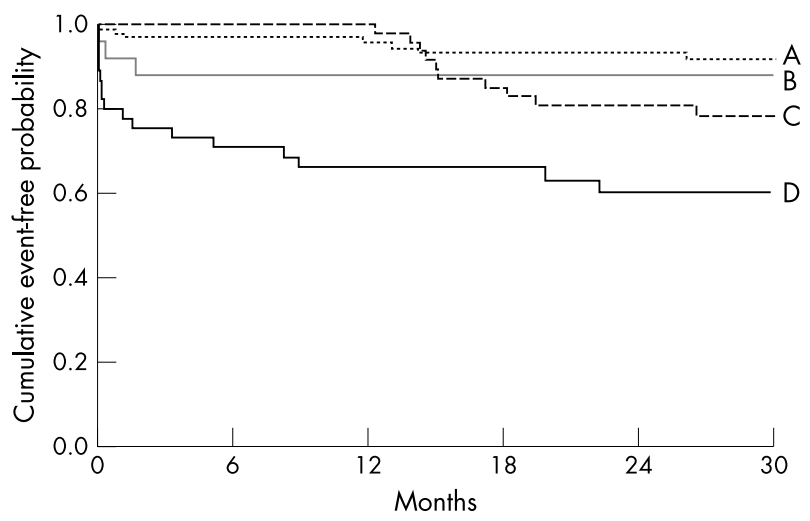

Figure 3 Occurrence of cardiac death or non-fatal AMl at a medium of 28 months' follow up in patients with troponin $\mathrm{T}<0.10 \mu \mathrm{g} / \mathrm{l}$ and no STVM episodes $(\mathrm{A}, \mathrm{n}=97)$, troponin $\mathrm{T}<0.10 \mu \mathrm{g} / \mathrm{l}$ and $\geqslant 1 \mathrm{ST}-\mathrm{VM}$ episodes $(B, n=24)$, troponin $T \geqslant 0.10 \mu \mathrm{g} / /$ and no ST-VM episodes ( $C, \mathrm{n}=47$ ), and troponin $\mathrm{T} \geqslant 0.10 \mu \mathrm{g} / \mathrm{l}$ and $\geqslant 1$ ST-VM episodes $(D, n=45)$. $A$ versus $B, \log$ rank $p=0.43 ; A$ versus $C, \log$ rank $p=0.09 ; A$ versus $D, \log$ rank $p=0.0001 ; B$ versus $C$, log rank $p=0.61 ; B$ versus $D$, log rank $p=0.02 ; C$ versus $D$, log rank $p=0.01$. 
Table 3 Multivariate stepwise Cox regression analysis of potential risk variables in order to detect independent predictors of cardiac death or AMI

\begin{tabular}{|c|c|c|c|c|}
\hline & \multicolumn{2}{|l|}{ Model I } & \multicolumn{2}{|l|}{ Model II } \\
\hline & Hazard ratio $(95 \% \mathrm{Cl})$ & p Value & Hazard ratio $(95 \% \mathrm{Cl})$ & p Value \\
\hline Age $\geqslant 65$ years & $1.06(1.02$ to 1.10$)$ & 0.005 & 1.05 (1.01 to 1.09$)$ & 0.01 \\
\hline Previous MI & 2.31 (1.13 to 4.73$)$ & 0.02 & $2.32(1.10$ to 4.86$)$ & 0.03 \\
\hline Previous revascularisation & $0.27(0.08$ to 0.94$)$ & 0.04 & $0.37(0.11$ to 1.32$)$ & 0.13 \\
\hline Congestive heart failure & 2.20 (1.04 to 4.67$)$ & 0.04 & $2.18(1.00$ to 4.75$)$ & 0.06 \\
\hline Admission use of $\beta$ blockers & $1.82(0.92$ to 3.59$)$ & 0.09 & $1.63(0.84$ to 3.22$)$ & 0.19 \\
\hline Troponin $T \geqslant 0.10 \mu \mathrm{g} / \mathrm{l}$ & NA & NA & $2.90(1.40$ to 6.00$)$ & 0.004 \\
\hline ST-VM $\geqslant 1$ episodes & NA & NA & 1.64 (0.84 to 3.22$)$ & 0.14 \\
\hline
\end{tabular}

Baseline variables of potential significance were evaluated (model I). Once variables in model I were accounted for, the added value of the information from troponin T testing and ST segment monitoring was explored (model II). All variables retained in the final models are shown.

$\mathrm{Cl}$, confidence interval; NA, not applicable.

Both troponin $\mathrm{T}$ and continuous multi-lead ST segment monitoring provide independent short term prognostic value in ACS. ${ }^{15}{ }^{16}$ Rather unexpectedly, the presence of transient ST episodes in the present study did not have significant long term prognostic value once the information from troponin testing was taken into account. During recent years it has been shown that patients at higher risk for a subsequent poor outcome as indicated by an increased troponin concentration or the presence of ST episodes benefit the most from a strategy of early angiography and revascularisation. ${ }^{19-21}$ In the present study $45 \%$ of the study population, on the discretion of the treating physicians, underwent revascularisation during follow up. During the TRIM study period, as recommended by the 1994 unstable angina guidelines, ${ }^{22}$ referral for angiography and revascularisation should be related to the patients risk of an adverse outcome such as the presence of recurrent angina and ischaemia at rest. Therefore, it may be speculated that the lack of blinding of the treating physicians to ST (in contrast to troponin) data in this study to some extent may have influenced treatment decisions (for example, referral for angiography), thus reducing the impact of ECG monitoring on late prognosis. However, we found no differences in frequency or timing of revascularisation according to the presence or absence of transient ST episodes. Moreover, the lack of late independent prognostic value of ECG monitoring remains valid even after sensitivity analysis with the censoring of patients at the time of coronary revascularisation. Thus, it is unlikely that the lack of blinding of the treating physicians to the ECG data in this study was a major determinant of the reduced impact of ECG monitoring on late prognosis.

\section{PATHOPHYSIOLOGICAL ASPECTS}

In ACS, plaque rupture or erosion results in a transient or persistent thrombotic vessel occlusion and transient fluctuations in the local coronary vasomotor tone. ${ }^{23}{ }^{24}$ The presence at coronary angiography in ACS of complex lesion morphology, severe coronary artery disease, or thrombus is associated with an unfavourable clinical outcome. ${ }^{10}{ }^{25-28}$ It has been shown that either an increased troponin concentration or the presence of transient ischaemic episodes in ACS is strongly associated with such angiographic findings, ${ }^{10} 2128-32$ thus explaining the prognostic value of troponin testing and ECG monitoring in ACS. The pathophysiological relation of troponin release and transient ischaemic episodes in ACS is further supported by the finding in this study of a positive quantitative association between the troponin $\mathrm{T}$ concentration and the ischaemic burden (as indicated by the number of ST episodes). Considering these concordant findings in patients with troponin increase and transient ischaemic episodes during the course of ACS, how can the different temporal risk characteristics of troponin testing and ECG monitoring as shown in this study be explained? Troponin increase in ACS probably reflects thrombus formation at the site of the plaque rupture and subsequent downstream embolisation of atherothrombotic material leading to minor myocardial necrosis. ${ }^{33}{ }^{34}$ In ACS, the size of thrombus changes with time ${ }^{33}$ and vasomotor reactivity is impaired, especially at the site of complex coronary lesions. ${ }^{24}$ Thus, it is conceivable that transient ischaemic episodes are caused by fluctuations in the size of thrombus or by transient vasospasms. On the basis of the findings in the present study it may be hypothesised that thrombus formation (as indicated by troponin increase) indicates a poor prognosis in ACS in both the short and the long term, whereas vasoconstriction (as indicated by the presence of transient ST episodes) at the most may have a role in predicting an unfavourable short term clinical course. This thesis is partially supported by the fact that in ACS only patients with troponin increase benefit from administration of antithrombotic (dalteparin) or antiplatelet (abciximab) agents. ${ }^{35} 36$ On the other hand, no prognostic benefit has been seen with vasospasmolytics, such as the nitrates, even though these agents reduce the ischaemic burden. ${ }^{37}$ Increasing troponin concentrations are associated with decreased left ventricular function, ${ }^{38}$ a pivotal determinant of mortality. ${ }^{39}$ In a study of patients with first time AMI, Rao and colleagues ${ }^{38}$ showed that a troponin $\mathrm{T}$ concentration $>2.8 \mu \mathrm{g} / \mathrm{l}$ predicted a left ventricular ejection fraction $<40 \%$ with a specificity of $92.9 \%$ (and sensitivity of $100 \%)$. In the present study, the upper quartile among patients with an increased troponin concentration was $1.90 \mu \mathrm{g} / \mathrm{l}$, thus left ventricular dysfunction was presumably not a major determinant for the prognostic value of troponin $\mathrm{T}$. Accordingly, troponin $\mathrm{T}$ increase in this study was related primarily to an increased risk of subsequent myocardial (re)infarction, and not to mortality.

\section{STUDY LIMITATIONS}

The present study population was selected according to the inclusion and exclusion criteria of the TRIM trial. The TRIM trial recruited patients from 1994 to $1995,{ }^{17}$ before the era of routine use of glycoprotein IIb/IIIa antagonists and early revascularisation of patients with ACS. The significance of immediate access of the local physicians to both troponin $\mathrm{T}$ and ST trend data for decision making needs to be delineated in future prospective studies. We may have underestimated the prognostic value of 12 lead ECG ST depression because we did not take the degree of ST depression into consideration.

\section{CONCLUSIONS}

When combining troponin $\mathrm{T}$ and continuous ST segment monitoring for early assessment of long term prognosis in 
patients with ACS, only troponin $\mathrm{T}$ testing provides significant independent prognostic information. Transient myocardial ischaemic episodes seem to be especially predictive of early cardiac adverse events. Although both biochemical and continuous ECG markers reflect an increased risk in ACS, they exhibit different temporal risk characteristics.

\section{ACKNOWLEDGEMENTS}

We appreciate the skilful assistance from the staff of each participating centre. We thank statistician Lars Frison PhD for valuable assistance with the statistical analyses.

This study was supported by a grant from the Danish Heart Foundation, Copenhagen, Denmark (96-2-3-36-22426).

\section{Authors' affiliations}

B L Nørgaard, K Thygesen, J Ravkilde, Department of Medicine and Cardiology A, Aarhus University Hospital, Aarhus, Denmark K Andersen, P Abrahamsson, L Grip, M Dellborg, Department of Medicine and Cardiology, Sahlgrenska University Hospital, Göteborg, Sweden

\section{REFERENCES}

1 Wu AHB, Abbas SA, Green S, et al. Prognostic value of cardiac troponin T in unstable angina pectoris. Am J Cardiol 1995;76:970-2.

2 Ravkilde J, Nissen H, Hørder M, et al. Independent prognostic value of serum creatine kinase isoenzyme MB mass, cardiac troponin T and myosin light chain levels in suspected acute myocardial infarction. Analysis of 28 months follow-up in 196 patients. J Am Coll Cardiol 1995;25:574-81.

3 Ohman EM, Armstrong PW, Christenson RH, et al. Cardiac troponin T levels for risk stratification in acute myocardial ischemia. N Engl J Med 1996;335:1333-41

4 Stubbs $\mathrm{P}$, Collinson P, Mosely D, et al. Prognostic significance of admission troponin T concentrations in patients with myocardial infarction. Circulation 1996;94:1291-7.

5 Stubbs $\mathbf{P}$, Collinson P, Mosely D, et al. Prospective study of the role of cardiac troponin $T$ in patients admitted with unstable angina. BMJ 1996;313:262-4

6 Lüscher MS, Thygesen K, Ravkilde J, et al for the TRIM Study Group. Applicability of cardiac troponin T and I for early risk stratification in unstable coronary artery disease. Circulation 1997;96:2578-85.

7 Holmvang $L$, Lüscher MS, Clemmensen $P$, et al. Very early risk stratification using combined ECG and biochemical assessment in patients with unstable coronary artery disease (a thrombin inhibition in myocardial ischemia [TRIM] substudy). Circulation 1998;98:2004-9.

8 Lindahl B, Toss H, Siegbahn A, et al. Markers of myocardial damage and inflammation in relation to long-term mortality in unstable coronary artery disease. N Engl J Med 2000;343:1139-47.

9 Gottlieb SO, Weisfeldt ML, Ouyang P, et al. Silent ischemia predicts infarction and death during 2 year follow-up of unstable angina. J Am Coll Cardiol 1987; 10:756-60.

10 Langer A, Freeman MR, Armstrong PW. ST segment shift in unstable angina: pathophysiology and association with coronary anatomy and hospital outcome. J Am Coll Cardiol 1989;13:1495-502.

11 Romeo F, Rosano GM, Martuscelli E, et al. Unstable angina: role of silent ischemia and total ischemic time (silent+painful ischemia), a 6-year follow-up J Am Coll Cardiol 1992;19:1173-9.

12 Dellborg M, Malmberg K, Ryden L, et al. Dynamic on-line vectorcardiography improves and simplifies in hospital ischemia monitoring of patients with unstable angina. J Am Coll Cardiol 1995;26:1501-7.

13 Andersen K, Eriksson P, Dellborg M. Non-invasive risk stratification within 48 hours of hospital admission in patients with unstable coronary disease. Eur Heart J 1996; 18:780-8.

14 Patel DJ, Knight DR, Holdright D, et al. Long term prognosis in unstable angina: the importance of early risk stratification using continuous ST segment monitoring. Eur Heart J 1998; 19:240-9.

15 Nørgaard BL, Andersen K, Dellborg M, et al. Admission risk assessment by cardiac troponin $\mathrm{T}$ in unstable coronary artery disease: additional prognostic information from continuous ST segment monitoring. J Am Coll Cardiol 1999;33:1519-27.
16 Jernberg $T$, Lindahl B, Wallentin L. The combination of a continuous 12-lead ECG and troponin T: a valuable tool for risk stratification during the first 6 hours in patients with chest pain and non-diagnostic ECG. Eur Heart $J$ 2000;21:1464-72.

17 Anon. A low-molecular-weight, selective thrombin inhibitor, inogatran, versus heparin in unstable coronary artery disease in 1209 patients: a double blind, randomised, dose-finding study. Thrombin inhibition in myocardial ischemia (TRIM) study group. Eur Heart $\mathrm{J}$ 1997; 18:1416-25

18 Müller-Bardoff M, Hallermayer K, Schröder A, et al. Improved troponin T ELISA specific for cardiac troponin $\mathrm{T}$ isoform: assay development and analytical and clinical validation. Clin Chem 1997;43:458-66.

19 Anon. Invasive compared with non-invasive treatment in unstable coronaryartery disease: FRISC II prospective randomised multicentre study. Fragmin and fast revascularisation during instability in coronary artery disease investigators. Lancet 1999:354:708-15.

20 Morrow DA, Cannon CO, Rifai N, et al. Ability of minor elevations of troponins I and T to predict benefit from an early invasive strategy in patients with unstable angina and non-ST elevation myocardial infarction. JAMA 2001;286:2405-12.

21 Diderholm E, Andren B, Frostfeldt G, et al. ST depression in ECG at entry indicates severe coronary lesions and large benefits of an early invasive treatment strategy in unstable coronary artery disease. The FRISC II ECG substudy. Eur Heart J 2002;23:41-9.

22 Braunwald $\mathrm{E}$, Mark DB, Jones $\mathrm{RH}$, et al. Unstable angina: diagnosis and management. Clinical practice guideline number 10 [AHCPR publication no 94-0602]. Rockville: Agency for Health Care Policy and Research and the National Heart, Lung, and Blood Institute, Public Health Service, US Department of Health and Human Services, 1994.

23 Falk E, Shah PK, Fuster V. Coronary plaque disruption. Circulation 1995;92:657-71.

24 Bogaty P, Hackett D, Davies G, et al. Vasoreactivity of the culprit lesion in unstable angina. Circulation 1994;90:5-11

25 Capone G, Wolf NM, Meyer B, et al. Frequency of intracoronary filling defects by angiography in angina at rest. Am J Cardiol 1985;56:403-6.

26 Califf RM, Phillips HR 3rd, Hindman MC, et al. rognostic value of a coronary artery jeopardy score. J Am Coll Cardiol 1985;5:1055-63.

27 Freeman MR, Williams AE, Chisholm RJ, et al. Intracoronary thrombus and complex morphology in unstable angina: relation to timing of angiography and in-hospital cardiac events. Circulation 1989;80:17-23.

28 Bugiadini R, Pozzati A, Borghi A, et al. Angiographic morphology in unstable angina and its relation to transient myocardial ischemia and hospital outcome. Am J Cardiol 1991;67:460-4.

29 Heeschen C, van den Brand MJ, Hamm CW, et al. Angiographic findings in patients with refractory unstable angina according to troponin $\mathrm{T}$ status. Circulation 1999;104:1509-14.

30 Newby LK, Kaplan AL, Granger BB, et al. Comparison of cardiac troponin T versus creatine kinase-MB for risk stratification in a chest pain evaluation unit. Am J Cardiol 2000;85:801-5.

31 Lindahl B, Diderholm E, Lagerqvist B, et al. Mechanisms behind the prognostic value of troponin T in unstable coronary artery disease: a FRISC II study. J Am Coll Cardiol 2001;38:879-86.

32 Patel DJ, Gomma AH, Knight CJ, et al. Why is recurrent myocardial ischaemia a predictor of adverse outcome in unstable angina? An observational study of myocardial ischemia and its relation to coronary anatomy. Eur Heart J 2001 ;22:1991-6.

33 Falk E. Unstable angina with fatal outcome: dynamic coronary thrombosis leading to infarction and/or sudden death. Autopsy evidence of recurrent mural thrombosis with peripheral embolization culminating in total vascular occlusion. Circulation 1985;71:699-708.

34 Topol EJ, Yadav JS. Recognition of the importance of embolization in atherosclerotic vascular disease. Circulation 2000;101:570-80.

35 Lindahl B, Venge P, Wallentin L. Troponin T identifies patients with unstable coronary artery disease who benefit from long-term antithrombotic protection. J Am Coll Cardiol 1997;29:43-8.

36 Hamm CW, Heeschen C, Goldmann B, et al. Benefit of abciximab in patients with refractory unstable angina in relation to serum troponin $T$ levels. N Engl J Med 1999:340:1623-9.

37 Chai AU, Crawford MH. "Traditional" medical therapy in unstable angina: how important? How to use? Cardiol Clin 1999;17:359-72.

38 Rao AC, Collison PO, Canepa-Anson R, et al. Troponin T measurement after myocardial infarction can identify left ventricular ejection of less than $40 \%$. Heart 1998;80:223-5.

39 St John Sutton M, Pfeffer MA, Plappert T, et al. Quantitative two-dimensional echocardiographic measurements are major predictors of adverse cardiovascular events after acute myocardial infarction: the protective effects of captopril. Circulation 1994;89:68-75. 